\title{
Net zero-emission pathways reduce the physical and economic risks of climate change
}

\author{
Laurent Drouet $\oplus^{1 凶}$, Valentina Bosetti ${ }^{1,2}$, Simone A. Padoan ${ }^{3}$, Lara Aleluia Reis $\odot 1$, \\ Christoph Bertram ${ }^{(1)^{4}}$, Francesco Dalla Longa $\oplus^{5}$, Jacques Després $\oplus^{6}$, Johannes Emmerling ${ }^{(1}$, \\ Florian Fosse $\oplus^{6}$, Kostas Fragkiadakis $\oplus^{7}$, Stefan Frank $\oplus^{8}$, Oliver Fricko $\oplus^{8}$, Shinichiro Fujimori $\oplus^{8,9,10}$, \\ Mathijs Harmsen $\oplus^{11,12}$, Volker Krey $\oplus^{8}$, Ken Oshiro $\oplus^{9}$, Larissa P. Nogueira $\oplus^{5}$, Leonidas Paroussos $\oplus^{7}$, \\ Franziska Piontek $\oplus^{4}$, Keywan Riahi $\oplus^{8,13}$, Pedro R. R. Rochedo ${ }^{14}$, Roberto Schaeffer $\left({ }^{14}\right.$, \\ Jun'ya Takakura ${ }^{10}{ }^{10}$, Kaj-Ivar van der Wijst $\circledast^{11,12}$, Bob van der Zwaan $\oplus^{5,15,16}$, Detlef van Vuuren $\oplus^{11,12}$, \\ Zoi Vrontisi ${ }^{7}$, Matthias Weitzel ${ }^{6}{ }^{6}$, Behnam Zakeri $\odot^{8}$ and Massimo Tavoni ${ }^{1}{ }^{1,17}$
}

\begin{abstract}
Mitigation pathways exploring end-of-century temperature targets often entail temperature overshoot. Little is known about the additional climate risks generated by overshooting temperature. Here we assessed the benefits of limiting overshoot. We computed the probabilistic impacts for different warming targets and overshoot levels on the basis of an ensemble of integrated assessment models. We explored both physical and macroeconomic impacts, including persistent and non-persistent climate impacts. We found that temperature overshooting affects the likelihood of many critical physical impacts, such as those associated with heat extremes. Limiting overshoot reduces risk in the right tail of the distribution, in particular for low-temperature targets where larger overshoots arise as a way to lower short-term mitigation costs. We also showed how, after mid-century, overshoot leads to both higher mitigation costs and economic losses from the additional impacts. The study highlights the need to include climate risk analysis in low-carbon pathways.
\end{abstract}

M ultiple mitigation trajectories are consistent with climate stabilization ${ }^{1}$, which may lead to different climate change risks ${ }^{2,3}$. One important feature of the pathways is the extent to which temperature is allowed to temporarily exceed a given target, commonly known as 'overshoot. Given historical emissions, stringent long-term temperature targets, such as limiting the temperature increase to $1.5^{\circ} \mathrm{C}$ in 2100 , often entail temporary temperature exceedance to be compensated by net negative carbon emissions in the second half of the century ${ }^{4}$. These pathways are the outcome of Integrated Assessment Models (IAMs) constrained to meet fixed-year targets, often for $2100^{5-7}$. The extent of overshoot is a function of many variables defining how rapidly human systems can be transformed, including socioeconomic and technological progress variables. For example, the assumptions of bioenergy technologies with carbon dioxide capture and geologic storage vary substantially across models ${ }^{8}$. It is also rooted in the choice of normative parameters. For example, time discounting consistent with proper consideration of future generations reduces overshoot and reliance on carbon dioxide removal ${ }^{9}$. Finally, the overshoot might depend on the way scenarios are designed and executed ${ }^{10}$. To overcome some of the limitations of end-of-century target scenarios, a scenario design has been recently proposed. It caps the peak temperature reached during the century, limiting 'net zero' carbon emissions ${ }^{11}$.

One reason for the temperature overshoot is that, usually, cost-minimizing emission pathways don't account for the climate benefits associated with different temperature trajectories. Detailed process IAMs, such as those providing input to the IPCC ${ }^{12}$, are tools primarily designed for mitigation analysis. As such, they don't take into account that overshoot trajectories lead to worse heat extremes than no-overshoot trajectories ${ }^{13}$. Benefit-cost IAMs include climate impacts, but lack mitigation strategy details and focus solely on monetary impacts ${ }^{14}$. Thus, their capacity to evaluate the full trade-offs implied by different intertemporal mitigation trajectories compliant with given climate stabilization targets is limited. Still, recent benefit-cost studies have highlighted the economic inequality repercussions in low-temperature cases ${ }^{15}$. Here we combined mitigation pathways with a postprocessing analysis of both physical and economic climate impacts, employing advanced statistical approaches. We used a large set of scenarios generated by a multimodel ensemble of nine leading detailed process IAMs, which explore end-of-century budget scenarios (where overshoot is allowed) versus net zero emission constrained budget scenarios.

'RFF-CMCC European Institute of Economics and the Environment, Centro Euro-Mediterraneo sui Cambiamenti Climatici, Milan, Italy. ${ }^{2}$ Department of Economics and IGIER, Bocconi University, Milan, Italy. ${ }^{3}$ Department of Decision Sciences, Bocconi University of Milan and Centro Euro-Mediterraneo sui Cambiamenti Climatici (CMCC), Milan, Italy. ${ }^{4}$ Potsdam Institute for Climate Impact Research (PIK), Member of the Leibniz Association, Potsdam, Germany. ${ }^{5}$ TNO Energy Transition, Amsterdam, the Netherlands. ${ }^{6}$ European Commission, Joint Research Centre (JRC), Seville, Spain. ${ }^{7}$ E3Modelling, Athens, Greece. ${ }^{8}$ International Institute for Applied Systems Analysis (IIASA), Laxenburg, Austria. ${ }^{9}$ Department of Environmental Engineering, Kyoto University, Kyoto, Japan. ${ }^{10} \mathrm{National}$ Institute for Environmental Studies (NIES), Tsukuba, Japan. "PBL Netherlands Environmental Assessment Agency, The Hague, the Netherlands. ${ }^{12}$ Copernicus Institute for Sustainable Development, Utrecht University, Utrecht, the Netherlands. ${ }^{13} \mathrm{Graz}$ University of Technology, Graz, Austria. ${ }^{14} \mathrm{CENERGIA/COPPE}$, Universidade Federal do Rio de Janeiro, Rio de Janeiro, Brazil. ${ }^{15}$ University of Amsterdam, Amsterdam, the Netherlands. ${ }^{16}$ Johns Hopkins University, Bologna, Italy. ${ }^{17}$ Department of Management, Economics and Industrial Engineering, Politecnico di Milano, Milan, Italy.

凶e-mail: laurent.drouet@eiee.org 

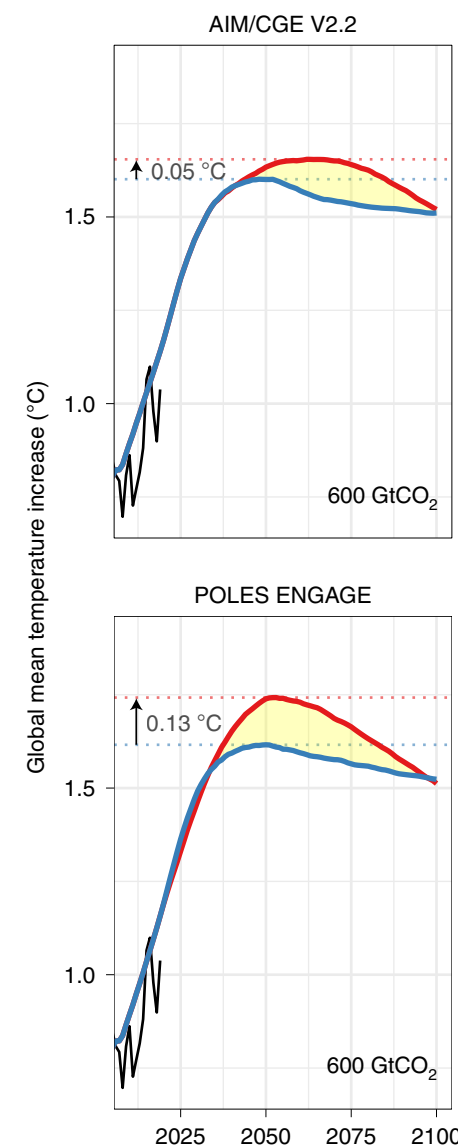

COFFEE 1.1
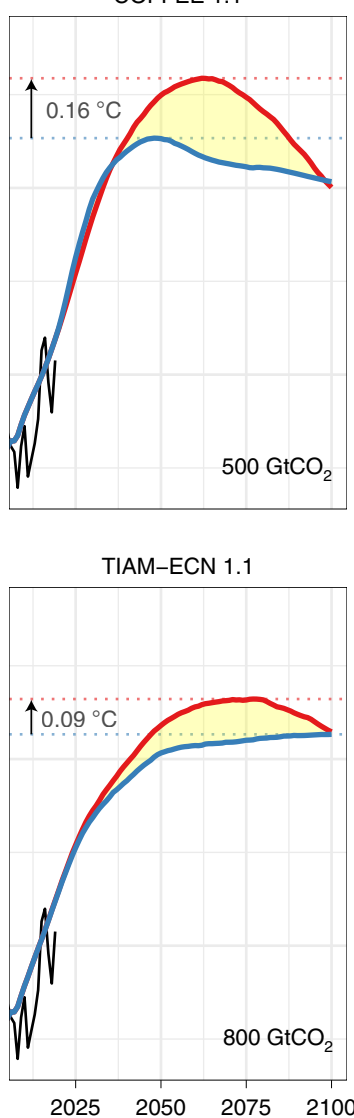

MESSAGEix-GLOBIOM_1.1

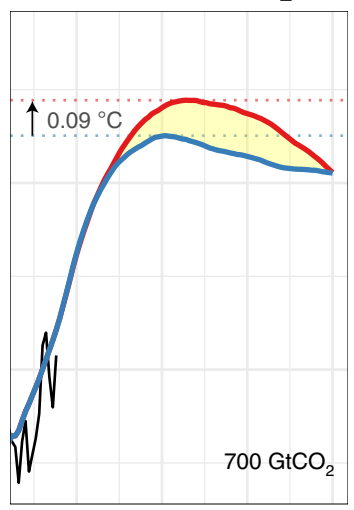

WITCH 5.0

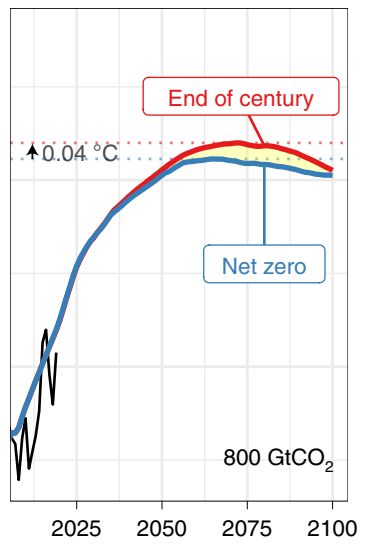

b
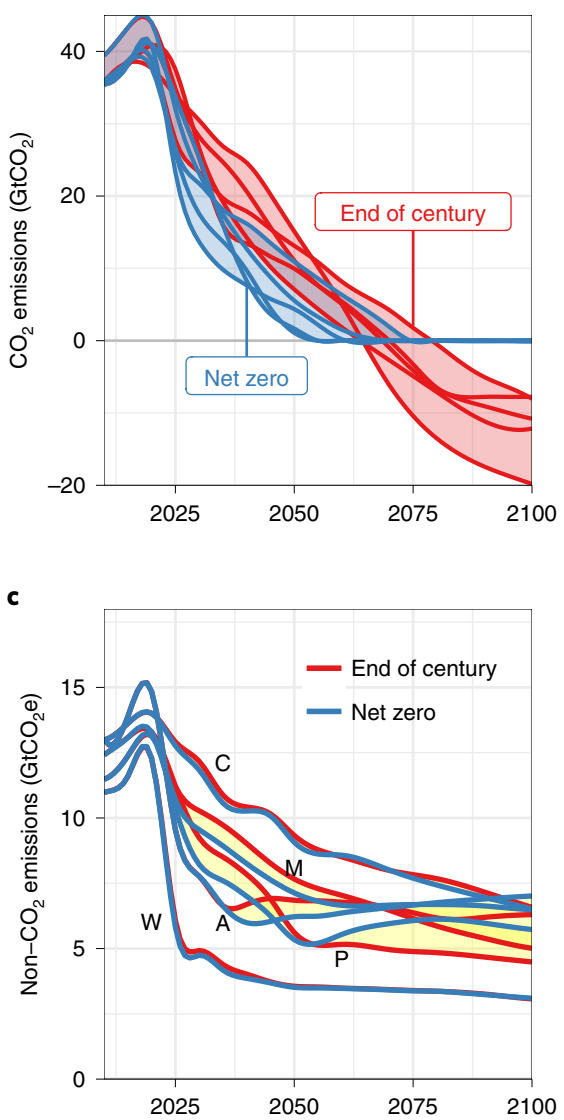

Fig. 1 | Influence of emission target formulation on the temperature and emission projections across models. a, The global mean temperature increase for an illustrative selection of model scenario combinations, leading to a similar temperature in 2100 , likely $1.5^{\circ} \mathrm{C}$. Each subpanel displays two scenarios for the same amount of cumulative emissions. The NZ design is in blue, and the EOC design is in red and allows for overshoot. The difference between the two trajectories is highlighted in yellow. Historical temperatures from HadCRUT4 (ref. ${ }^{35}$ ) are shown until 2019 by the black lines. The maximum temperatures of trajectories are indicated by horizontal dotted lines and their difference is shown beside each up arrow. $\mathbf{b}$, The global $\mathrm{CO}_{2}$ emission projections for each model and scenario design, highlighting the ranges. c, The global non- $\mathrm{CO}_{2}$ emission projections $\left(\mathrm{CH}_{4}, \mathrm{~N}_{2} \mathrm{O}\right.$ and f-gases), expressed as $\mathrm{CO}$-eq using $\mathrm{GWP}$ 100. Differences between EOC and NZ are highlighted. The letters are the models' initials. Extended Data Fig. 1 reports all temperature pathways of the cluster (likely $1.5^{\circ} \mathrm{C}$ ). Supplementary Fig. 28 provides model details for $\mathbf{b}$ and $\mathbf{c}$.

The pairwise comparison highlights the overshoot implications while reaching Paris Agreement compliant targets. We generated probabilistic climate outcomes from the scenario ensembles. We used the latest impact science to derive probabilistic climate impacts for a wide array of physical and economic indicators. Results show that the climate benefits of limiting overshoot can be important, especially for stringent climate targets with larger overshoot. The benefits occur for both physical and macroeconomic impacts, albeit interesting differences occur. Limiting overshoot is effective in reducing low-probability high-consequence climate change repercussions.

This study is part of a multimodel comparison exercise, which also focuses on the near-term energy system investments ${ }^{16}$, the mitigation costs of overshooting ${ }^{17}$, and on the land-use sector ${ }^{18}$.

\section{Scenario protocol}

This study involves nine global integrated assessment models: AIM/ CGE, COFFEE, GEM-E3, IMAGE, MESSAGEix-GLOBIOM,POLES, REMIND-MAgPIE, TIAM-ECN and WITCH (Supplementary Information). These models have been widely used to assess global climate change mitigation pathways ${ }^{6,7,19}$. They are representative of a wide spectrum of approaches, spanning from simulation to optimization models, and from game-theoric frameworks to least-cost optimization models. They all have a detailed representation of the energy and land-use systems and a wide array of decarbonization options. Used in conjunction, the models generate an ensemble of pathways that span a plausible range of technological developments, allowing us to assess the results' robustness and highlight trajectories characterized by fat tail risk where the likelihood of high impact is much greater than that of a normal distribution.

Each modelling team followed the same protocol to ensure comparative results (Supplementary Methods). After 2020, the models impose a remaining carbon budget, that is, the cumulative $\mathrm{CO}_{2}$ emissions over the period 2018-2100, consistent with a given long-term temperature target, for two different scenario designs. The 'End of Century' (EOC) scenario design implements the remaining carbon budget without restriction, while the 'Net Zero' (NZ) scenario design implements the remaining carbon budget until $\mathrm{CO}_{2}$ emissions reach net zero $\mathrm{CO}_{2}$ emissions. After that point, net $\mathrm{CO}_{2}$ emissions are kept at zero. This ensures that the temperature peaks and stabilizes. The emission pathways are produced for a large range of remaining carbon budgets, from $500 \mathrm{GtCO}_{2}$ to $2,000 \mathrm{GtCO}_{2}$, to explore the space of climate targets systematically ${ }^{20}$. Models constrain their cumulative $\mathrm{CO}_{2}$ emissions and price the other greenhouse gases emissions 
Table 1 | Global geophysical maximum impacts over the century. The table reports the median values, the 5th and 95th percentiles (in brackets) of the distributions of maximum impacts over the century for the NZ and the EOC, for two temperature clusters. The impact indicator definitions are provided in Supplementary Table 5

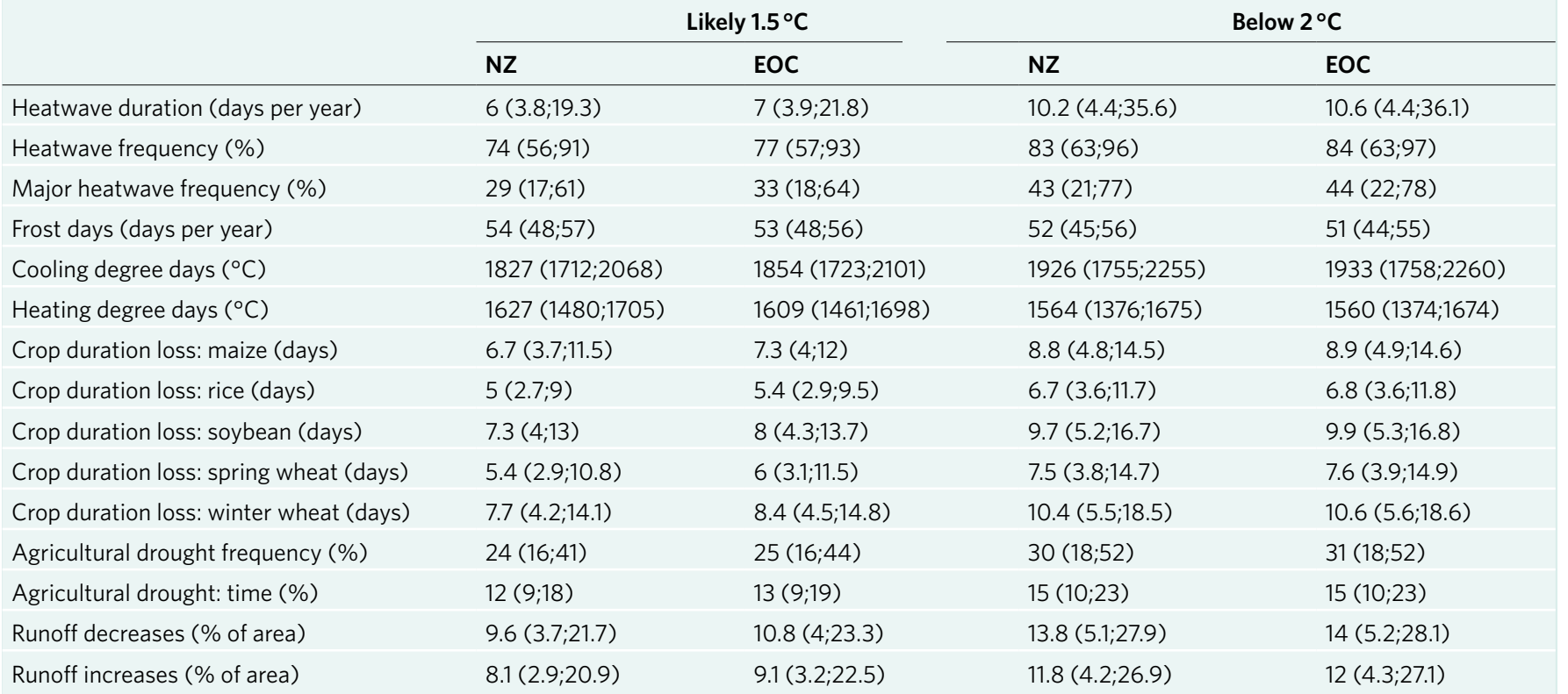

at the $\mathrm{CO}_{2}$ price adjusted by the 100 -year global warming potential equivalent. We compared the pair of trajectories produced by each model for the same remaining carbon budget (Fig. 1).

We used the climate emulator MAGICC ${ }^{21,22}$ to project the global mean temperature consistently across scenarios, in a setup reproducing the IPCC AR5 climate sensitivity uncertainty assessment ${ }^{23,24}$. EOC and NZ scenarios under the same budget lead to nearly the same temperature increase in 2100, but the timing and the level of the warming peak vary across scenario designs and across models (Fig. 1a). The IAMs do not directly control the overshoot magnitude, which instead depends on the model structure and its mitigation options. EOC scenarios rely heavily on negative $\mathrm{CO}_{2}$ emissions at the end of the century, while $\mathrm{NZ}$ scenarios reduce $\mathrm{CO}_{2}$ emissions earlier and to lower levels (Fig. 1b and Supplementary Fig. 22). As a consequence, peak warming happens earlier and is lower in the NZ scenario than in the EOC scenario (Fig. 1a and Supplementary Fig. 1).

The cumulated temperature overshoot, that is, the cumulated difference in temperature between EOC and NZ along the century's path (yellow area in Fig. 1a), depends on the model but also on the remaining carbon budget: the more stringent the remaining carbon budget and hence the lower the temperature increase in 2100, the larger the overshoot, robustly across all models (Supplementary Fig. 2). Similarly, the maximum overshoot, that is, the temperature difference between the maximum temperature in EOC and NZ, declines with larger remaining carbon budgets, although model differences exist (Supplementary Fig. 3). These temperature trajectories suggest that we could expect the largest climate impact differentials between EOC and NZ scenarios with smaller remaining carbon budgets.

Across models, the same remaining carbon budget may not necessarily lead to the same 2100 temperature, as the models reduce the non- $\mathrm{CO}_{2}$ greenhouse gases emissions differently (Fig. 1c). As temperatures represent our key input to impact calculations, we clustered the model carbon budget pairs (EOC and NZ) according to the temperature reached in 2100 . We characterized the scenarios as: likely $1.5^{\circ} \mathrm{C}$ for a temperature of $1.55^{\circ} \mathrm{C}$ in 2100 , likely $1.6^{\circ} \mathrm{C}$ for a temperature between $1.55^{\circ} \mathrm{C}$ and $1.65^{\circ} \mathrm{C}$, below $1.8^{\circ} \mathrm{C}$ for a temperature between $1.65^{\circ} \mathrm{C}$ and $1.8^{\circ} \mathrm{C}$, and below $2^{\circ} \mathrm{C}$ for a temperature between $1.8^{\circ} \mathrm{C}$ and $2{ }^{\circ} \mathrm{C}$. The temperature clusters contained a similar number of scenarios for a diversity of models and temperature trajectories (Supplementary Table 1 and Figs. 1 and 23).

\section{Physical impact distribution}

We started off by gauging the probabilistic climate implications of a wide array of physical indicators on the basis of regional impact functions, also representing the uncertainty in the regional pattern of climate change from the models of the fifth phase of the Coupled Model Intercomparison Project (CMIP5) ${ }^{25}$. The temperature distributions coming from the range of IAM scenarios of the comparison exercise were translated into physical impact distributions for each temperature cluster and scenarios. We produced the distributions for different impacts: heat extremes (four different indicators), energy demand (two), agriculture (seven) and water resources (two) at both the global and regional scale.

The maximum geophysical impact over the century can help to compare the effect of the scenario design (Table 1 and Supplementary Figs. 4 and 6). The most striking difference was observed between likely $1.5^{\circ} \mathrm{C}$ and below $2{ }^{\circ} \mathrm{C}$, where the median values increased for all impacts, showing the high sensitivity of impacts to temperature ${ }^{2,4}$. The differences in impacts related to heatwaves were among the most severe. The regions most affected by the changes in heatwaves are Brazil, West and Southern Africa (Supplementary Fig. 7).

We explored the role of temperature overshoot, comparing the characteristics of the impact distributions. The differences in median impacts between EOC and NZ scenarios were modest but still statistically indistinguishable (under the $90 \%$ confidence interval); for example, overshoot increased the median of maximum heatwave duration by $1 \mathrm{~d}$ for likely $1.5^{\circ} \mathrm{C}$ scenarios and $0.4 \mathrm{~d}$ for below $2{ }^{\circ} \mathrm{C}$ scenarios (see also Fig. 2a). In addition, the maximum impacts were always lower in NZ scenarios, consistent with the temperature trajectories. The same conclusions held at the regional level, but with a different ranking for the various impacts (Supplementary Table 2).

We compared the distributions of the maximum impact occurring over the century, with and without overshoot at likely $1.5^{\circ} \mathrm{C}$, using a Kolmogorov-Smirnov test (Supplementary Fig. 5). Globally, 

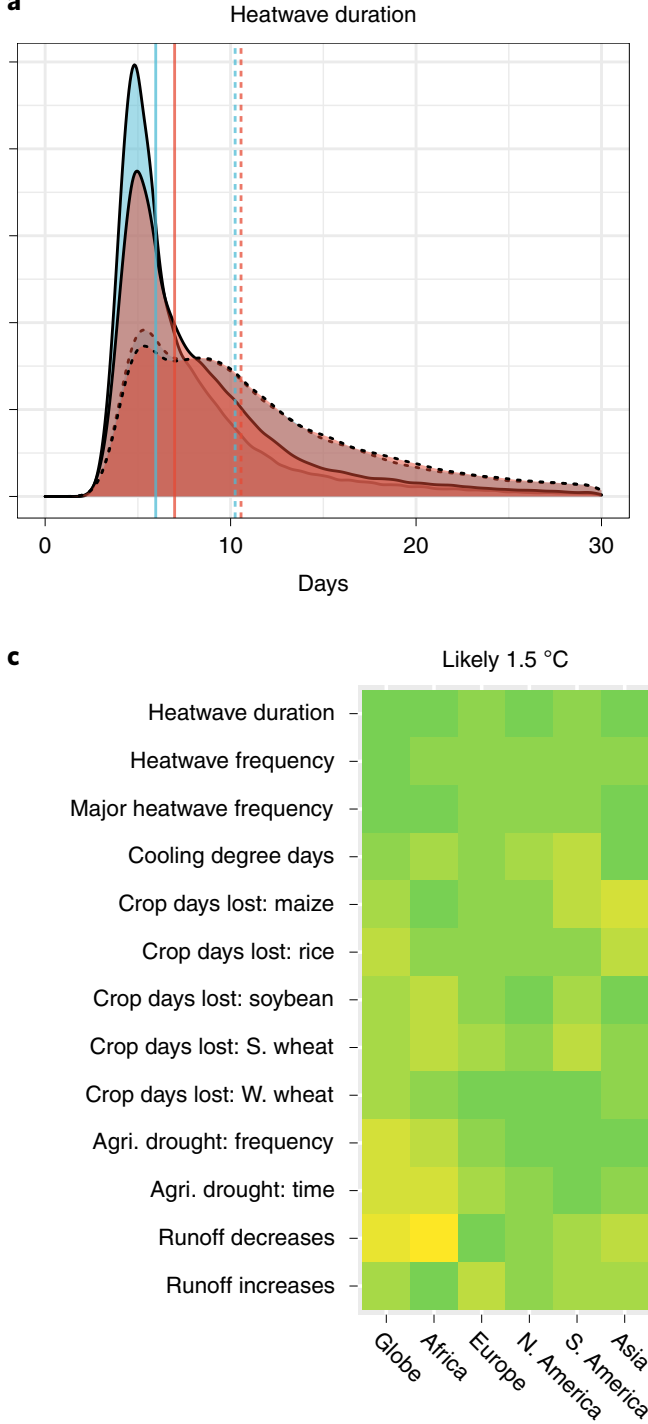
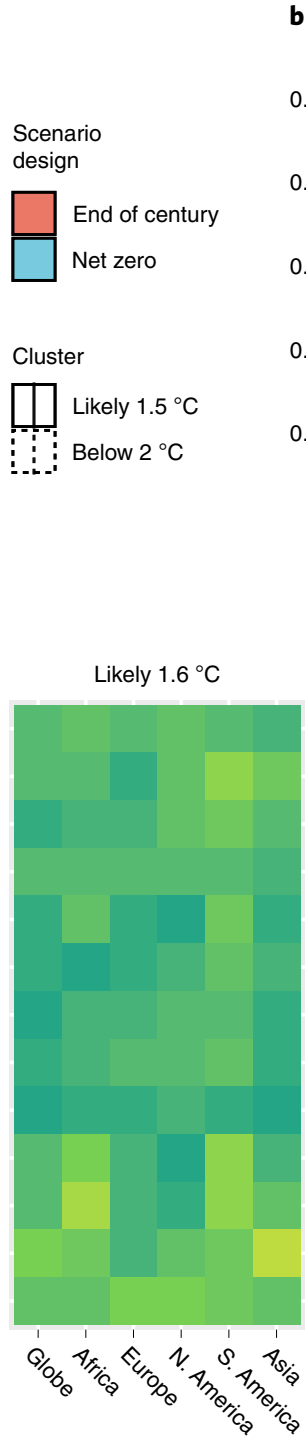

b
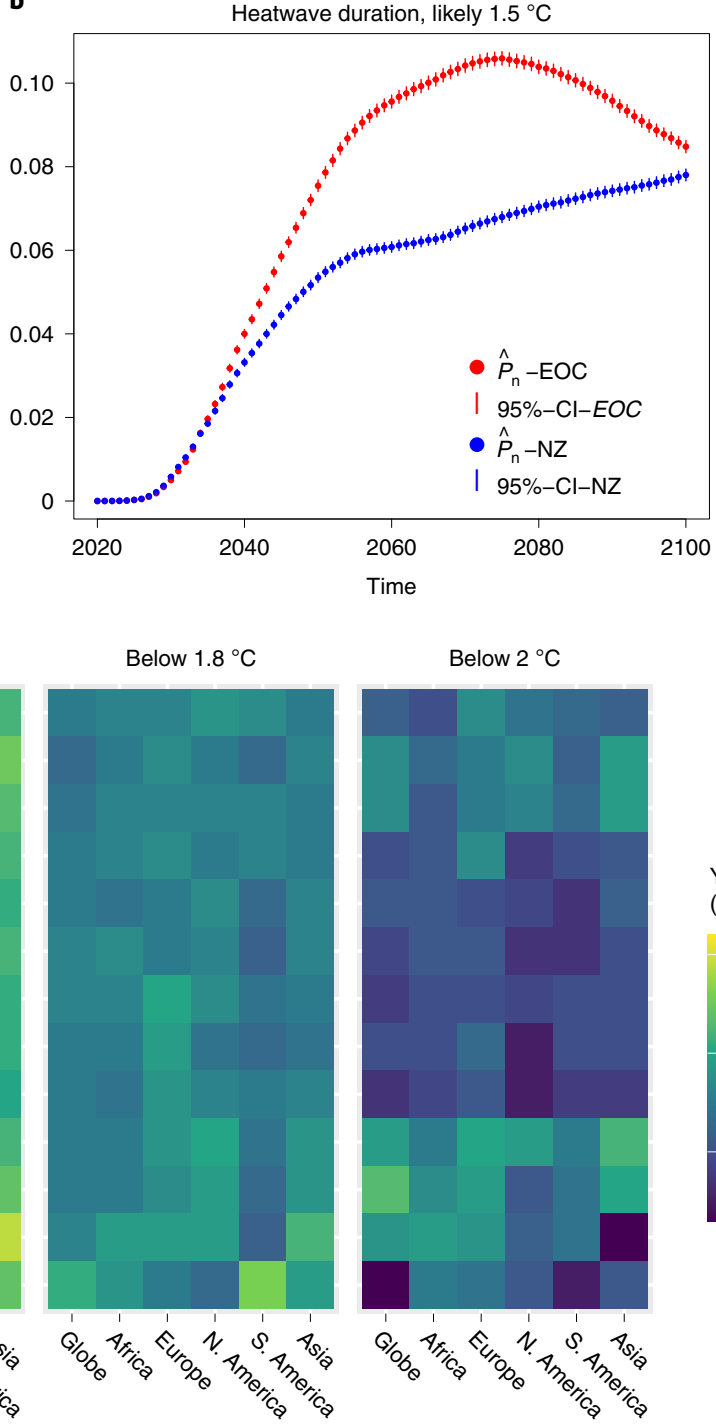

Years $(\mathrm{EOC}>\mathrm{NZ})$

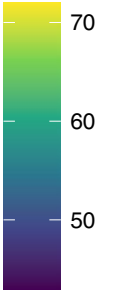

Fig. 2 | Influence of the scenario design on the impact distribution. a, The probability density of the maximum heatwave duration over the century, expressed in days per year. The colour indicates the scenario design. Linetype distinguishes the temperature clusters. The vertical line shows the median. b. Plots of the probability of exceeding the threshold over time for heatwave duration, for the likely $1.5^{\circ} \mathrm{C}$ cluster. The threshold is defined as heatwave duration over the century in the NZ scenario at the 95th quantile ( $=15 \mathrm{~d}$ ). The segments represent the $95 \%$ confidence intervals of the probability of exceeding the threshold. The colour indicates the scenario design. c, The number of years where the impact tail distribution in the EOC scenario is longer than that in the NZ scenario. Impacts are displayed along the $y$ axis and regions are along the $x$ axis. The subpanels correspond to the four temperature clusters. In all panels, the sources of uncertainty are the scenarios, the climate sensitivity and the CMIP5 model impact response (Supplementary Fig. 14).

the most notable climate benefits of reduced overshoot were the ones related to growth crop duration (in particular, for rice and winter wheat) and energy demands (cooling degree days and heating degree days). At the regional level, impacts related to heatwaves would most likely be reduced in Africa and North America. In Europe, crop duration would be the most likely to be affected for maize, soybean, winter wheat and rice.

The impacts related to heatwaves are the most critical ones in terms of sensitivity to temperature change, even at low temperatures $^{25,26}$. The extra burden imposed by allowing the temperature to overshoot can be appreciated when comparing the maximum impacts in the NZ and EOC scenarios (Fig. 2a). The implications of overshooting are most visible at low-temperature levels. Tail events or high-consequence low-probability events have often been used to justify stringent action in the face of climate change ${ }^{27}$.
For all impacts, we explored the probability of exceeding 'high' values and then performed empirical tests to determine whether the exceeding probabilities differ across NZ and EOC scenarios. In our case, as we are lacking observations, the 'high' value is the median of the impacts in the NZ scenario over the century. While the median might not be a high threshold for a given year, it is for some specific years and it helps in understanding how the exceeding probability evolves across years. For example, the probability of exceeding 5.02 $\mathrm{d}$ of heatwave in the EOC scenario is significantly higher than in the $\mathrm{NZ}$ scenario after 2040 in the likely $1.5^{\circ} \mathrm{C}$ cluster (Fig. 2b). Similar analyses were systematically conducted across impact indicators and regions (Supplementary Fig. 13).

For several impacts and regions under the likely $1.5^{\circ} \mathrm{C}$ cluster, overshooting might mean 60 years of higher exceeding probability (Fig. 2c). The number of years with significant differences due to overshooting decreases when the temperature target increases, but 
a

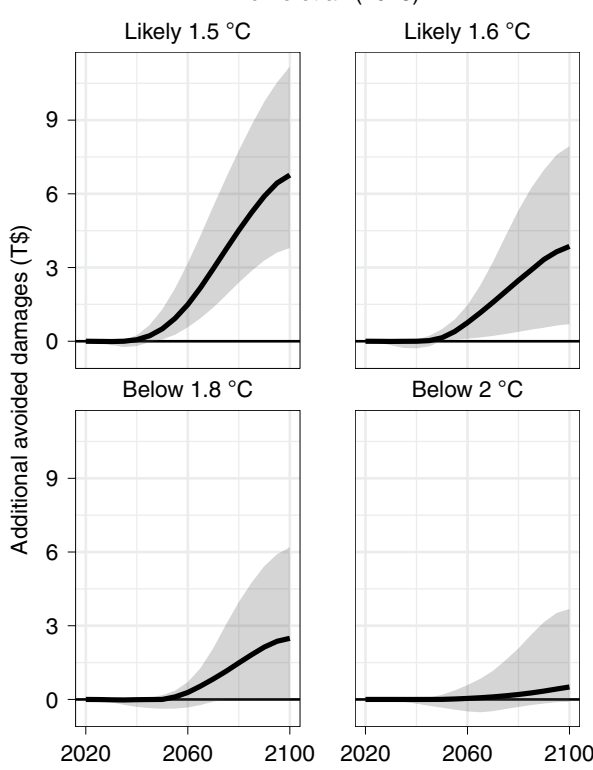

b

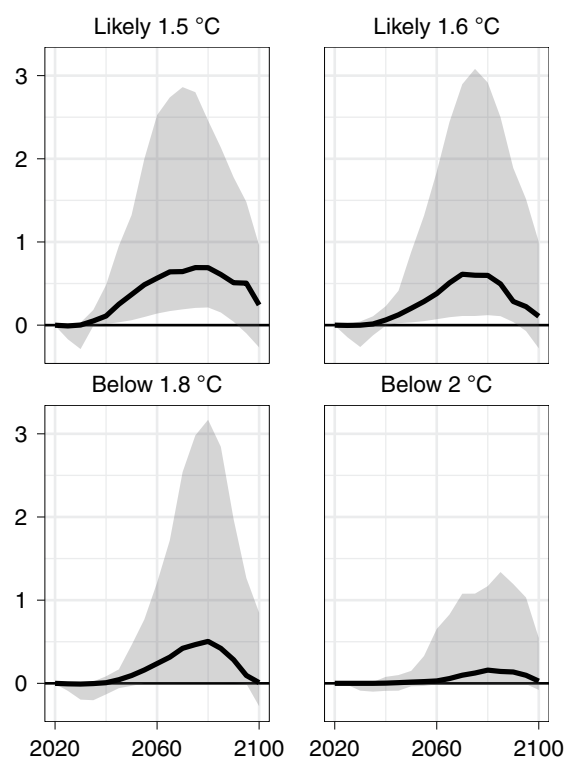

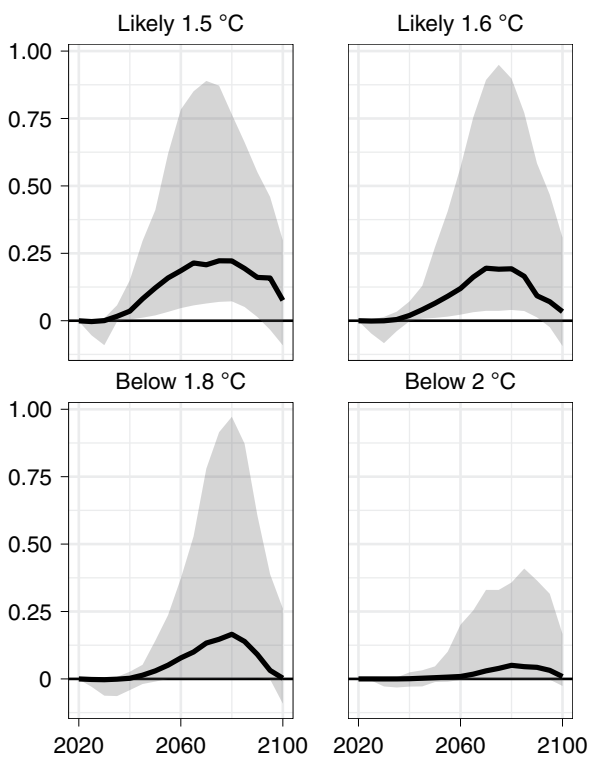

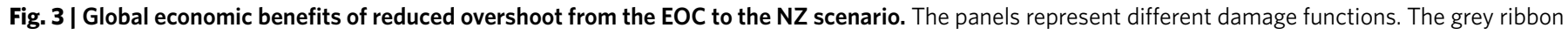
shows the 5-95\% range. The black line is the median. Uncertainty sources are the scenarios, the climate sensitivity (for all panels) and the CMIP5 model pattern (for a).

there are some disparities across indicators (extreme heat and crop duration are the most at risk) and across regions (Africa is the most at risk with South America, while Europe and North America are less at risk).

Finally, we projected sea-level rise from the temperature projections to look at the potential benefits of NZ scenarios on impacts based on cumulative warming. Over the period 2020-2200, the global mean sea-level rise increase due to temperature overshooting alone is $0.4-2.3 \mathrm{~cm}$ at likely $1.5^{\circ} \mathrm{C}$, while the increase under the $\mathrm{NZ}$ scenario for the same temperature cluster is $75 \mathrm{~cm}(57-109 \mathrm{~cm})$ (Supplementary Figs. 8 and 9). Thus, temporary temperature exceedance in Paris compliant scenarios does not have a substantial impact on sea-level rise, given the uncertainties surrounding it.

\section{Economic impacts}

An increasingly rich literature explores the long-term implications of climate change on the macroeconomy ${ }^{28-30}$. The estimates from this literature vary widely depending on the methods used and the underlying assumptions. There are obvious limits in any exercise aiming at monetization of climate damage, and for this reason, the economic assessment should only serve as a way to complement the physical impact analysis. However, detailed physical impact assessment is limited to selected channels and misses additional repercussions rippling through the economy, for example, from interactions, feedbacks and exacerbations through repeated events. Therefore, it is critical to use macroeconomic estimates capturing general equilibrium effects and using sectorial damage functions. The existing macroeconomic damage estimates differ primarily on the extent to which economic damages persist, and whether climate shocks are assumed to affect the growth rate or the level of the economy ${ }^{29,31}$. To represent this diversity, we used a set of damage functions that encompass both approaches, on the basis of recent literature.

First, we relied on empirical estimates of the relation between temperature variations and gross domestic product (GDP) growth ${ }^{32}$. For this, we downscaled the global mean temperature change to the country level and applied the warming effect to country-level GDP projections. In this case, climate change impacts are persistent over time and observed additional adaptation is accounted for ${ }^{32}$. Secondly, we applied a quadratic damage function, calibrated on the most recent estimates of global impacts from climate change ${ }^{33}$, that reduces the GDP level, with non-persistent damages. Finally, we considered another quadratic damage function, reproducing the estimates computed by a general equilibrium model including regional and sectorial damage functions ${ }^{34}$. The three damage functions include no or few accountings of non-market damages and probably underestimate the total economic impact.

NZ scenarios brought more climate economic benefits or avoided more damage than EOC scenarios, independently of the temperature clusters or the damage function (Fig. 3). The median climate economic benefits accrue over time and are higher for more stringent temperature targets, since the avoided overshoot is larger in these cases. The growth versus level damage functions yield qualitatively and quantitatively different results. For growth-based estimates, the avoided impacts are larger and increase in absolute terms over time because of the persistence of the benefit of lower transient temperatures. For level-based functions, the climate benefits of reduced overshoot are mostly transient and vanish by the end of the century as temperatures converge.

From an economic point of view, comparing NZ and EOC scenarios is not trivial, as it implies comparing different intertemporal mitigation profiles as well as the avoided impacts by overshooting. The flexibility to smooth the mitigation effort across time implied by EOC scenarios reduces the short-term cost of otherwise more rapid decarbonization, but it concurrently increases the risks. The costbenefit trade-off in favour of limiting temperature overshoot evolves over time for those scenarios for which the overshoot is larger, at likely $1.5^{\circ} \mathrm{C}$ (Fig. 4). The timing and magnitude of net benefits are driven by the type of damage function. In all cases, scenarios with limited overshoot yield benefits after 2050 both in terms of reduced climate impact and lowered mitigation costs (Supplementary Figs. 10 and 12). The net present value of the stream of benefits and costs for the different scenarios (Supplementary Fig. 30) shows larger net present benefits than costs for two of the three damage functions, for all temperature clusters (although this difference is not always 


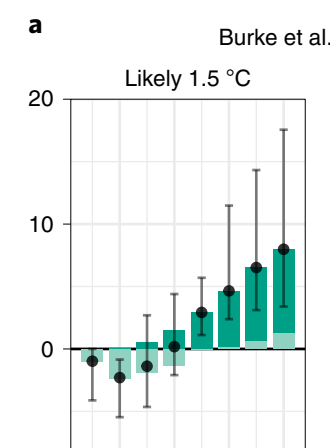

$\stackrel{\overparen{E}}{E}$

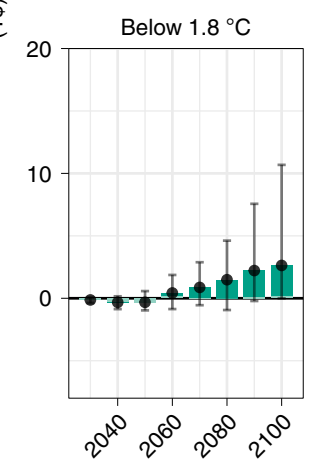

(2015)

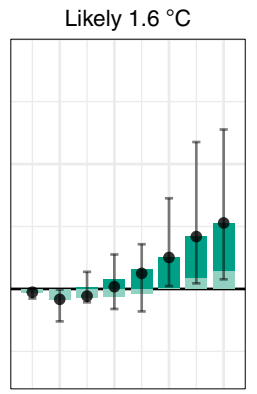

Below $2{ }^{\circ} \mathrm{C}$

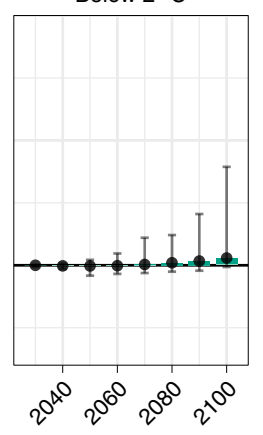

b

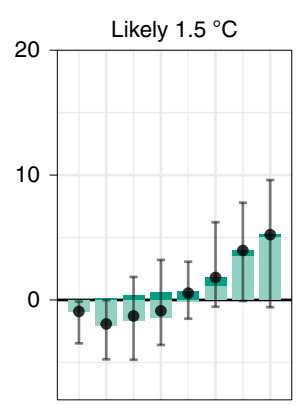

Below $1.8^{\circ} \mathrm{C}$

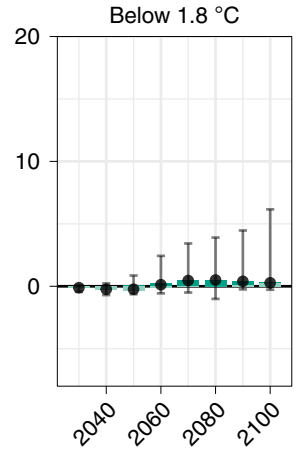

17)

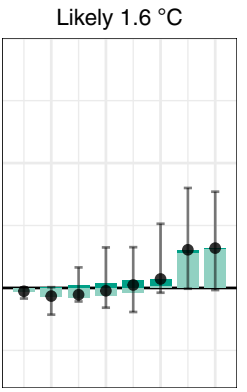

Below $2{ }^{\circ} \mathrm{C}$

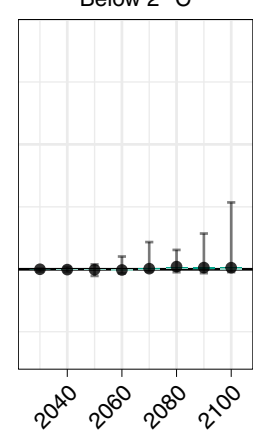

C

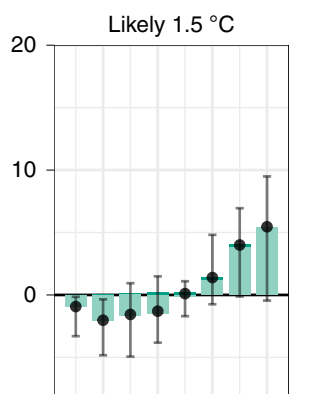

Below $1.8^{\circ} \mathrm{C}$

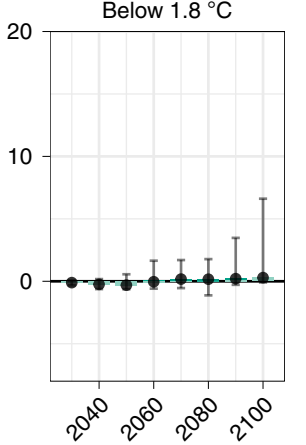

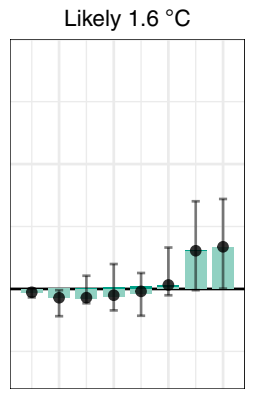

Below $2{ }^{\circ} \mathrm{C}$

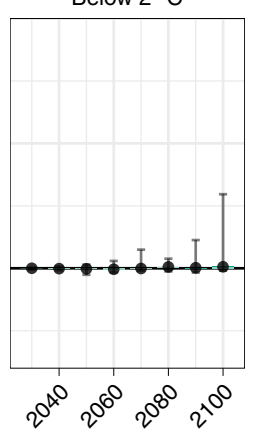

Benefits (above 0) and costs (below 0) from

damages and

Fig. 4 | Global economic benefits and mitigation costs of reduced overshoot between the EOC and the NZ scenarios. The panels represent different damage functions. The dark green bars and the light green bars show the median of the additional avoided damages and the median of the reduction in mitigation costs, respectively. The errors bars show the 5-95\% range. Uncertainty sources are the scenarios, the climate sensitivity (for all panels) and the CMIP5 model pattern (for a).

statistically distinguishable from zero). In the case of the impact model in ref. ${ }^{34}$, the net present value is negative for all temperature clusters, but this result also cannot be statistically distinguished from zero. The net present value of the difference between the two scenario designs is reported in Supplementary Fig. 29: only for the two lowest temperature clusters and for the impact model based on ref. ${ }^{32}$ did the no-overshooting policy design pass the net present value test. For all other cases, the two policy designs cannot be discriminated on the basis of this test.

\section{Discussion}

The analysis presented here has explored the physical and macroeconomic impacts associated with mitigation pathways with different levels of temperature overshoot.

This work provides a novel bridge between the detailed process assessment of mitigation pathways, which typically explores the costs and risks associated with climate transition, and the climate impact community, which investigates the costs and risks of climate change.

The results confirm the centrality of intertemporal and risk preferences when assessing alternative mitigation strategies. Limiting temperature overshoot by anticipating mitigation efforts leads to a stream of climate change benefits, cuts the right tail of the distribution of different impact indicators and eventually lowers mitigation costs. All these benefits accrue during the second half of the century. Therefore, the choice of the discount rate, as well as preferences over these extreme risks, determine whether overshooting can be considered as a viable option or not.

Economic assessments of avoided climate damage alone might overlook some of the physical impacts we might face in the future, reinforcing the need to complement economic analyses with work on physical impact estimates.
Finally, exploring the different economic impact models and assumptions (for example, damage persistence), we found that early mitigation costs are never significantly larger than the climate benefits of not overshooting.

\section{Online content}

Any methods, additional references, Nature Research reporting summaries, source data, extended data, supplementary information, acknowledgements, peer review information; details of author contributions and competing interests; and statements of data and code availability are available at https://doi.org/10.1038/ s41558-021-01218-z.

Received: 12 December 2020; Accepted: 10 October 2021;

Published online: 29 November 2021

\section{References}

1. Seneviratne, S. I. et al. The many possible climates from the Paris Agreement's aim of $1.5^{\circ} \mathrm{C}$ warming. Nature 558, 41-49 (2018).

2. O'Neill, B. C. \& Oppenheimer, M. Climate change impacts are sensitive to the concentration stabilization path. Proc. Natl Acad. Sci. USA 101, 16411-16416 (2004).

3. Schneider, S. H. \& Mastrandrea, M. D. Probabilistic assessment of 'dangerous' climate change and emissions pathways. Proc. Natl Acad. Sci. USA 102, 15728-15735 (2005).

4. Hoegh-Guldberg, O. et al. in IPCC Special Report: Global Warming of $1.5^{\circ} \mathrm{C}$ (eds Masson-Delmotte, V. et al.) Ch. 3 (IPCC, 2018).

5. Rogelj, J. et al. Scenarios towards limiting global mean temperature increase below $1.5^{\circ} \mathrm{C}$. Nat. Clim. Change 8, 325-332 (2018).

6. Luderer, G. et al. Residual fossil $\mathrm{CO}_{2}$ emissions in $1.5-2^{\circ} \mathrm{C}$ pathways. Nat Clim. Change 8, 626-633 (2018).

7. Vrontisi, Z. et al. Enhancing global climate policy ambition towards a $1.5^{\circ} \mathrm{C}$ stabilization: a short-term multi-model assessment. Environ. Res. Lett. 13 044039 (2018). 
8. Daioglou, V. et al. Bioenergy technologies in long-run climate change mitigation: results from the EMF-33 study. Clim. Change 163, 1603-1620 (2020).

9. Emmerling, J. et al. The role of the discount rate for emission pathways and negative emissions. Environ. Res. Lett. 14, 104008 (2019).

10. Johansson, D. J. A., Azar, C., Lehtveer, M. \& Peters, G. P. The role of negative carbon emissions in reaching the Paris climate targets: the impact of target formulation in integrated assessment models. Environ. Res. Lett. 15, 124024 (2020)

11. Rogelj, J. et al. A new scenario logic for the Paris Agreement long-term temperature goal. Nature 573, 357-363 (2019).

12. Clarke, L. et al. in Climate Change 2014: Mitigation of Climate Change (eds Edenhofer, O. et al.) Ch. 6 (Cambridge Univ. Press, 2014).

13. Sanderson, B. M. et al. Community climate simulations to assess avoided impacts in $1.5^{\circ} \mathrm{C}$ and $2^{\circ} \mathrm{C}$ futures. Earth Syst. Dynam. 8, 827-847 (2017).

14. Pezzey, J. C. V. Why the social cost of carbon will always be disputed. WIREs Clim. Change 10, e558 (2019).

15. Gazzotti, P. et al. Persistent inequality in economically optimal climate policies. Nat. Commun. 12, 3421 (2021).

16. Bertram, C. et al. Energy system developments and investments in the decisive decade for the Paris Agreement goals. Environ. Res. Lett. 16, 074020 (2021).

17. Riahi, K. et al. Implications of avoiding temperature overshoot for stringent mitigation pathways. Nat. Clim. Change https://doi.org/10.1038/s41558-02101215-2 (2021).

18. Hasegawa, T. et al. Land-based implications of early climate actions without global net-negative emissions. Nat. Sustain. https://doi.org/10.1038/ s41893-021-00772-w (2021).

19. Roelfsema, M. et al. Taking stock of national climate policies to evaluate implementation of the Paris Agreement. Nat. Commun. 11, 2096 (2020).

20. Fujimori, S., Rogelj, J., Krey, V. \& Riahi, K. A new generation of emissions scenarios should cover blind spots in the carbon budget space. Nat. Clim. Change 9, 798-800 (2019).

21. Meinshausen, $\mathrm{M}$. et al. Greenhouse-gas emission targets for limiting global warming to $2^{\circ} \mathrm{C}$. Nature 458, 1158-1162 (2009).

22. Meinshausen, M., Raper, S. C. B. \& Wigley, T. M. L. Emulating coupled atmosphere-ocean and carbon cycle models with a simpler model, MAGICC6 - Part 1: model description and calibration. Atmos. Chem. Phys. 11, 1417-1456 (2011).
23. Rogelj, J., Meinshausen, M. \& Knutti, R. Global warming under old and new scenarios using IPCC climate sensitivity range estimates. Nat. Clim. Change 2, 248-253 (2012)

24. Rogelj, J., Meinshausen, M., Sedláček, J. \& Knutti, R. Implications of potentially lower climate sensitivity on climate projections and policy. Environ. Res. Lett. 9, 031003 (2014).

25. Arnell, N. W., Lowe, J. A., Challinor, A. J. \& Osborn, T. J. Global and regional impacts of climate change at different levels of global temperature increase. Clim. Change 155, 377-391 (2019).

26. Lange, S. et al. Projecting exposure to extreme climate impact events across six event categories and three spatial scales. Earths Future 8 e2020EF001616 (2020)

27. Weitzman, M. L. Fat-tailed uncertainty in the economics of catastrophic climate change. Rev. Environ. Econ. Policy 5, 2 (2011).

28. Moore, F. C. \& Diaz, D. B. Temperature impacts on economic growth warrant stringent mitigation policy. Nat. Clim. Change 5, 127-131 (2015).

29. Piontek, F. et al. Economic growth effects of alternative climate change impact channels in economic modeling. Environ. Resour. Econ. 73, 1357-1385 (2019).

30. Tol, R. S. J. et al. The economic impacts of climate change. Rev. Environ. Econ. Policy 12, 4-25 (2018).

31. Guivarch, C. \& Pottier, A. Climate damage on production or on growth: what impact on the social cost of carbon? Environ. Model. Assess. 23, 117-130 (2018).

32. Burke, M., Hsiang, S. M. \& Miguel, E. Global non-linear effect of temperature on economic production. Nature 527, 235-239 (2015).

33. Howard, P. H. \& Sterner, T. Few and not so far between: a meta-analysis of climate damage estimates. Environ. Res. Econ. 68, 97-225 (2017).

34. Takakura, J. et al. Dependence of economic impacts of climate change on anthropogenically directed pathways. Nat. Clim. Change 9, 737-741 (2019).

35. Morice, C. P., Kennedy, J. J., Rayner, N. A. \& Jones, P. D. Quantifying uncertainties in global and regional temperature change using an ensemble of observational estimates: the HadCRUT4 data set. J. Geophys. Res. Atmos. 9, 737-741 (2012).

Publisher's note Springer Nature remains neutral with regard to jurisdictional claims in published maps and institutional affiliations.

(C) The Author(s), under exclusive licence to Springer Nature Limited 2021 


\section{Methods}

Model scenarios. For this analysis, we used the results from nine integrated assessment models: AIM/CGE, COFFEE, GEM-E3, IMAGE, TIAM-ECN, POLES, REMIND-MAgPIE, MESSAGEix-GLOBIOM and WITCH. These models generated emission trajectories consistent with a large number of carbon budgets (that is, cumulative $\mathrm{CO}_{2}$ emissions) using two families of scenarios: EOC, which allowed for temperature overshoot, and NZ, which did not (see the modelling protocol in Supplementary Information). Models are not constrained to the level of temperature over the century and it can overshoot the temperature reached in 2100 . We selected the scenarios entailing maximum warming of $2{ }^{\circ} \mathrm{C}$ in 2100 . The protocol included a vetting exercise to ensure that model results were sufficiently close to historical data up to 2020. In particular, attention was devoted to harmonizing the energy system (that is, installed power plant capacities, investments and activities), greenhouse gases and aerosol emissions, the land-use sector and economic growth. Models also implemented policies in force, such as carbon taxes, constraints on fossil fuels, renewables standards, etc. The protocol did not require capturing the effect of the COVID-19 pandemic. A comprehensive description of the modelling process and the model scenarios is provided by ${ }^{17}$.

Uncertainty analysis. Various sources of uncertainty were considered in the analysis: the emission trajectories to reach a carbon budget, the global mean temperature through the climate sensitivity, and the CMIP5 model patterns in terms of geophysical impact response and country-level temperature. Supplementary Fig. 14 lists these sources of uncertainty and provides a representation of the uncertainty propagation.

Global mean temperature. The emissions pathways from all model scenarios were given as an input to the climate emulator MAGICC ${ }^{21,22}$ to compute global mean temperature projections until 2100 (median estimate, 5\%, 10\%, 25\%, 75\%, 90\% and $95 \%$ quantiles). MAGICC was calibrated to represent the climate sensitivity uncertainty assessed in the IPCC special report on the impacts of global warming of $1.5^{\circ} \mathrm{C}$. The model scenarios were clustered on the basis of the median of the 2100 global mean temperature (Supplementary Table 1).

Temperature downscaling. Country-level annual population-weighted temperature projections were obtained from the median estimates of the global mean temperature using a linear response function calibrated for each CMIP5 model. We gathered monthly mean temperatures from historical data records and the RCP runs of 20 CMIP5 models with all available ensemble members (ACCESS1-0 (3 runs), BNU-ESM (4), CCSM4 (29), CMCC-CMS (3), GFDL-CM3 (8), GFDL-ESM2G (5), GFDL-ESM2M (5), GISS-E2-H (15), GISS-E2-H-CC (3), GISS-E2-R (15), GISS-E2-R-CC (3), HadGEM2-CC (7), HadGEM2-ES (19), IPSL-CM5A-LR (19), IPSL-CM5A-MR (7), IPSL-CM5B-LR (2), MPI-ESM-LR (12), MPI-ESM-MR (8), NorESM1-M (7) and inmcm4 (3)). We computed the gridded annual mean temperature and corrected the bias using a 1980-2016 observational baseline (Supplementary Fig. 21). Unbiased gridded annual mean temperatures were aggregated at the country level with population density weights based on the gridded population of the world in 2010 (GPW v4). Results are comparable with the original baselines from ref. ${ }^{32}$ (see sensitivity analyses in Supplementary Figs. 26 and 27). Finally, to obtain an estimate of the annual local temperature, from the global mean temperature increase relative to 2005, we performed a linear regression over the period 1900-2100 for each CMIP5 model for each year and each country individually.

Physical impacts. For each model scenario, we computed 15 impact indicators (see the list and definition in Supplementary Table 5) every year for 6 regions (global and 5 macro regions: Africa, Europe, North America, South America, Asia). The physical impacts were computed from a look-up table of global and regional impacts of climate change at different levels of global temperature increase, differentiated for 23 CMIP5 climate models ${ }^{25}$. To apply those functions, the global mean temperature was shifted down by $0.014^{\circ} \mathrm{C}$ so that the average temperature increase relative to the pre-industrial level is equal to $0.61^{\circ} \mathrm{C}$ over the period 1981-2010 to replicate ref. ${ }^{25}$. The impacts of intermediate temperatures were interpolated linearly. Linear interpolation provides better consistency across the impact functions (Supplementary Fig. 17). We also evaluated the spline interpolation, which resulted in some values being out of credible bounds (for example, negative values) for a few combinations of temperature and impact (Supplementary Fig. 18). The difference between the two methods of interpolation is much smaller than the impact values (Supplementary Figs. 19 and 20). The impact distribution results from the combination of the model scenarios, the global mean temperature distribution and the CMIP5-specific impact function. Using these distributions, yearly values and maximum over-thecentury comparisons were performed for the impact analyses. Note that our study focuses on the transient response of climate and impacts, which cannot be fully captured by simple pattern scaling techniques ${ }^{36}$. However, this is the best available method that allows us to capture the uncertainties stemming from consistent impact estimates spanning 5 levels of warming and 23 climate patterns ${ }^{25}$.
Economic impacts using the growth-based damage function. The economic impacts were computed at the country level. We followed the procedure described in ref. ${ }^{32}$ and implemented in ref. ${ }^{37,38}$. GDP per capita is $G_{i, t}=G_{i, t-1}\left(1+\eta_{i, t}+\delta\left(T_{i, t}\right)\right)$, where $\eta_{i}$ is the growth rate coming from the Shared Socioeconomic Pathways (SSP) reference projection in which no climate change occurs ${ }^{39}$ and $\delta\left(T_{i, t}\right)$ is a response function of the temperature increase at year $t$. The projected warming effect was adjusted by the baseline temperature in 2000-2010. The analysis used the main damage function specification called $\mathrm{BHM} \mathrm{SR}^{32}$.

Economic impacts from the level-based damage functions. These economic impacts were only computed at the global level. They were computed as the global output loss relative to the SSP reference projection without climate change (GDP_gross). The GDP loss is $\Delta G D P \_c c=G D P \_$gross $\times\left(\alpha g m t_{t}+\beta g m t_{t}^{2}+\gamma\right)$, where gmt is the global mean temperature increase from pre-industrial levels and $\alpha$ and $\beta$ are the two parameters of the quadratic damage function. For the Howard \& Sterner function ${ }^{33}$, we used the preferred model specification of non-catastrophic damage, which was increased by $25 \%$ to account for the omitted damage in the empirical estimates $(\alpha=\gamma=0, \beta=-0.7438)$. For the Takakura et al. function ${ }^{34}$, we derived and used the SSP 2 function parameters $(\alpha=0.07625, \beta=0.21465$, $\gamma=-0.11746)$.

Tail heaviness analysis. We performed a statistical analysis to test whether the EOC distribution has a longer tail than the NZ distribution. The assumptions and the methodology for the tail heaviness analysis are provided in detail in Supplementary Methods.

Sea-level rise. We computed the global mean sea-level rise using the physical model provided by ref. ${ }^{40}$, using their calibration. For this specific impact, we extended the time horizon of the computations until 2200, with a constant global mean temperature beyond 2100 . Sea levels keep rising through the twenty-second century. We computed the sea-level rise for 3 quantiles (5\%, 50\% and 95\%).

Avoided damages and mitigation costs. The additional damages associated with the overshooting of the temperature target were obtained by comparing GDP in the EOC and the NZ scenarios, when impacts from climate change were accounted for in both scenarios (Supplementary Fig. 11). Depending on the model characteristics, the proxy for mitigation costs used was either GDP losses or the additional energy system cost calculated with respect to a reference scenario where only policies in force are considered - the 'NPi2100' in the modelling protocol (Supplementary Methods). To ensure consistency across impact and mitigation costs, all economic values were expressed in USD2018 using the reference GDP projection.

\section{Data availability}

The global climate change mitigation scenario dataset analysed in this study is available in Zenodo (https://doi.org/10.5281/zenodo.5553976). The future GDP per capita are from https://tntcat.iiasa.ac.at/SspDb/dsd. The historical gridded temperature is from the University of Delaware air temperature UDEL v5.01 dataset (https://psl.noaa.gov/data/gridded/data.UDel_Air_Precip.html). The gridded population of the world in 2010 can be obtained from https://sedac.ciesin. columbia.edu/data/collection/gpw-v4.

\section{Code availability}

The repository of the source code used for the data analysis and the figures can be found at https://github.com/lolow/ENGAGE-overshoot-impacts. The code version to reproduce this study is available at https://doi.org/10.5281/zenodo.5555380.

\section{References}

36. King, A. D., Lane, T. P., Henley, B. J. \& Brown, J. R. Global and regional impacts differ between transient and equilibrium warmer worlds. Nat. Clim. Change 10, 42-47 (2020).

37. Ricke, K., Drouet, L., Caldeira, K. \& Tavoni, M. Country-level social cost of carbon. Nat. Clim. Change 8, 895-900 (2018).

38. Burke, M., Davis, W. M. \& Diffenbaugh, N. S. Large potential reduction in economic damages under UN mitigation targets. Nature 557, 549-553 (2018).

39. Riahi, K. et al. The shared socioeconomic pathways and their energy, land use, and greenhouse gas emissions implications: an overview. Glob. Environ. Change 42, 153-168 (2017).

40. Li, C., Held, H., Hokamp, S. \& Marotzke, J. Optimal temperature overshoot profile found by limiting global sea level rise as a lower-cost climate target. Sci. Adv. https://doi.org/10.1126/sciadv.aaw9490 (2020).

\section{Acknowledgements}

We thank H. Held for discussion on the sea-level rise model. This research received funding from the European Union's Horizon 2020 research and innovation programme under grant agreement no. 821471 (ENGAGE) (L.D., V.B, L.A.R, C.B., F.D.L., J.D., J.E., F.F., S. Frank, K.F., O.F., S. Fujimori, M.H., V.K., L.P.N., K.O., L.P., F.P., R.S., J.T., K.R., 
P.R.R.R, D.v.V., M.T., Z.V., M.W., K.-I.v.d.W, B.Z. and B.v.d.Z). S. Fujimori is supported by the Environment Research and Technology Development Fund (JPMEERF20202002) of the Environmental Restoration and Conservation Agency of Japan and by the Sumitomo Foundation.

\section{Author contributions}

L.D. and V.B. designed the research, with contributions from K.R.; L.D., V.B, L.A.R, C.B., F.D.L., J.D., J.E., F.F., S. Frank, K.F., O.F., S. Fujimori, M.H., V.K., L.P.N., K.O., L.P., F.P., R.S., J.T., K.R., P.R.R.R, D.v.V., M.T., Z.V., M.W., K.-I.v.d.W, B.Z. and B.v.d.Z produced the IAM scenario results; L.D. postprocessed the data and performed the data analysis; S.P. performed the statistical analysis; L.D., V.B. and M.T. wrote the paper draft; L.D., V.B., J.E, M.T. and L.A.R. finalized the manuscript, with contributions from F.P, R.S, J.T. and M.W.; all authors reviewed the manuscript.

\section{Competing interests}

The authors declare no competing interests.

\section{Additional information}

Extended data is available for this paper at https://doi.org/10.1038/s41558-021-01218-z.

Supplementary information The online version contains supplementary material available at https://doi.org/10.1038/s41558-021-01218-z.

Correspondence and requests for materials should be addressed to Laurent Drouet.

Peer review information Nature Climate Change thanks James Rising and the other, anonymous, reviewer(s) for their contribution to the peer review of this work.

Reprints and permissions information is available at www.nature.com/reprints. 
a) likely $1.5^{\circ} \mathrm{C}$

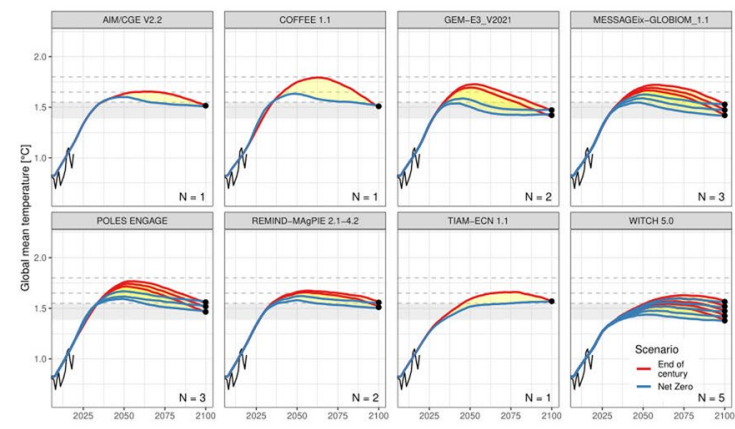

b) likely $1.6^{\circ} \mathrm{C}$

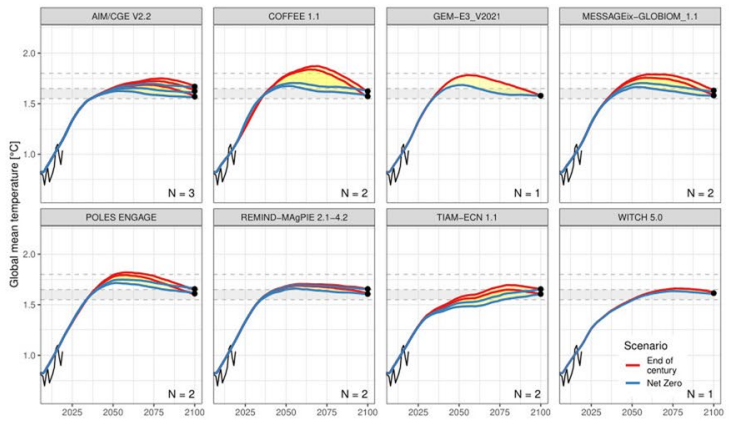

c) below $1.8^{\circ} \mathrm{C}$

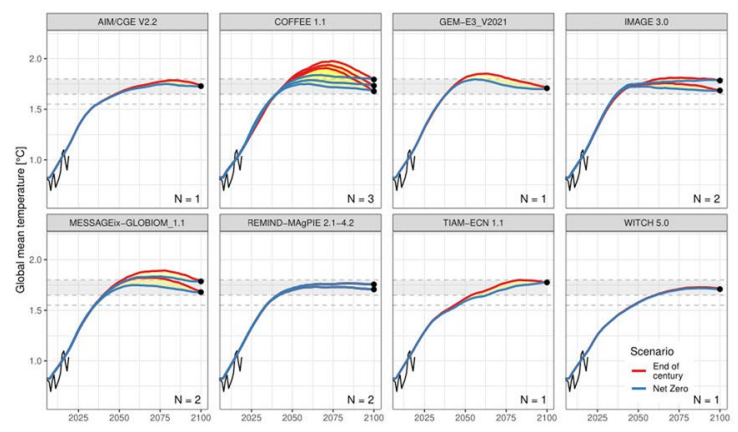

d) below $2^{\circ} \mathrm{C}$

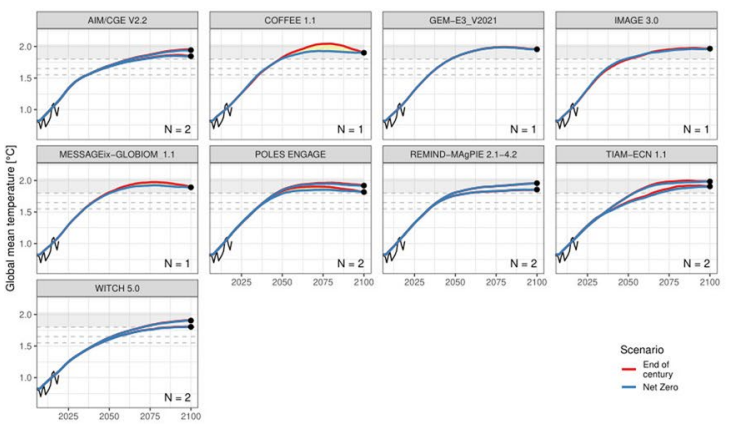

Extended Data Fig. 1 | Influence of the temperature cluster and the model scenario on the global mean temperature. Each subpanel shows pairs of scenarios leading to the same temperature in 2100. The number of pair scenarios is denoted in the bottom right. The color distinguished the scenario design. 'Net Zero' (NZ) design is in blue, and the 'End of Century' (EOC) design is in red. The temperature difference is highlighted in yellow. Historical temperature are shown in black. 